\title{
Distributed Fault Detection Using Consensus of Markov Chains
}

\author{
Dejan P. Jovanović* and Philip K. Pollett*
}

\begin{abstract}
We propose a fault detection procedure appropriate for use in a variety of industrial engineering contexts, which employs consensus among a group of agents about the state of a system. Markov chains are used to model subsystem behaviour, and consensus is reached by way of an iterative method based on estimates of a mixture of the transition matrices of these chains. To deal with the case where system states cannot be observed directly, we extended the procedure to accommodate Hidden Markov Models.
\end{abstract}

\section{Index Terms}

Fault detection, consensus algorithm, mixtures of Markov chains, the EM algorithm, Hidden Markov Model (HMM), multi-agent systems.

\section{INTRODUCTION}

With the development of new types of engineering systems, such robotic networks and networks of unmanned aerial vehicles (UAVs), and initiatives to modernize already established engineering systems such as "smart" power grids, the problem of fault detection is becoming more import, because early detection of deviations in system characteristics from the norm leads to increased reliability and maintainability [1]. Early detection is achieved by building local behavioural models and establishing information exchange protocols to estimate local characteristics for decentralized decision making.

Theoretical background on fault detection is part of the wider milieu of procedures for recognizing unwanted behaviour in monitored systems. Fault diagnosis generally comprises

*Department of Mathematics, University of Queensland, Qld 4072 AUSTRALIA. Email: dejan.jovanovic@uqconnect.edu.au

*Department of Mathematics, University of Queensland, Qld 4072 AUSTRALIA. Email: pkp@maths.uq.edu.au 
three sequential steps (known as the fundamental tasks of fault diagnosis [2]). The first, fault detection, is to decide whether the characteristics of the system in question are outside permissible limits. The second, fault identification, is to determine which subsystems contain a fault of a particular type and the time when it occurred. Finally, fault analysis provides insight into the time-varying characteristics of the fault and the scale of disturbance that occurred.

We focus here primarily on fault detection. In order to identify change, we need an adequate reference model for system features. This is the backbone of the model-based fault detection approach ([3], [4], [5], [2], [6]) adopted here. Three common methods used in feature generation within the context of model-based fault detection are parameter estimation ([7], [8]), state estimation ([3], [9]) and parity (consistency) checking [5]. Once estimated, the present state is compared with that of nominal (normal) system behaviour and a residual is generated that measures any change. In model-based fault detection the full set of residuals is used for decision making, and a change in their mean and/or covariance signals a fault [6].

The choice of model depends on the problem at hand. However, it is often impractical to build a model for the entire system. This is particularly true for distributed systems, where there are many interrelated and interconnected parts. A natural approach is to simplify the task by first decomposing the system into a number of subsystems, which would usually be spatially separated and assumed to evolve independently. The decomposition may be deterministic [10] or probabilistic [11]. Attached to each subsystem is a set of independent local observations and a set of local parametric models that describe different working conditions. We will used the term "agent" as an abstraction that integrates these two components. A group decision is accomplished through interaction between neighbouring agents, and one of our goals is determine the conditions for logical (behavioural) consensus [12] among group members.

A state-space model is a common option for many practical problems in fault detection [9], and a Kalman estimator [13] is frequently used to estimate the mean and covariance of the state. However, the latter approach suffers from a lack of robustness to noise and uncertainties [6]. Our approach is different. Instead of generating residuals, we estimate the probability distribution of the state from the given observations. This is compared with corresponding distribution in the normal mode. The "distance" between these distributions 
is measured in order to decide on the presence of a fault. We assume that under any given operating mode, be it normal or faulty, the state of any subsystem can be described faithfully by a discrete-state Markov chain [14]. Normal operating conditions are described by a single model and there are a number of models indicating a fault. We assume each agent can accurately detect faults in its own subsystem.

Achieving consensus by arriving at a common distribution representing belief among agents is an idea that goes back to the early sixties, when Stone [15] introduced pooling of opinions to determine a group decision. Stone assumed that each opinion was modelled by a continuous probability distribution, and the opinion pool as a mixture of distributions. However, he considered only equal mixture weights. DeGroot [16] extended this idea in two ways: first, by introducing an iterative approach whereby each agent revises its own subjective probability distribution based on changes in other group members opinions, and, second, by allowing the possibility of distinct weights and specifying convergence conditions for the iteration. The convergence conditions were generalized further by Chatterjee and Seneta [17]. We follow DeGroot's approach, but extend this using Markov chains rather than continuous distributions. In our method, agents reach consensus about the transition matrices that govern changes in subsystem state within each operating mode. At each iteration agent $i$ revises its own transition matrix by taking a weighted sum of the other agents' matrices and its own. The weights are selected optimally using an ExpectationMaximization (EM) framework. As we shall see, our method extends easily to the case of hidden Markov chains, thus allowing for states that may not be directly observable.

Once consensus is achieved, fault diagnosis can commence. To decide whether there is a fault, each agent compares its local distribution with a consensus distribution, being the stationary distributions of the local and the consensus transition matrices, respectively. If the "distance" between these distributions is greater than 0, a fault is recorded. Once it has been established that there are faulty subsystems, the next step is to determine which subsystems are faulty and what type of fault is present. There are two scenarios. In the first, a subsystem identifies its own fault and checks if there are group members affected by the same fault (the agent compares its own local model with other local models in the group by measuring the distance between corresponding stationary distributions). In the second, if the subsystem has no fault then the faulty subsystems can be detected in the same manner 
by again comparing stationary distributions. A range of different distance values indicates multiple faults.

We note that a consensus algorithm has been used in the work of Franco et al. [18], Ferrari et al. [19] and Stanković et al. [20]. It differs from ours in that first and second order moments only were used, rather than the entire distribution. We note also that Petri

Nets have been used extensively in fault detection in distributed systems [21]. Particularly interesting is the work of Benveniste et al. [22], who introduced a probabilistic extension of Petri Nets for distributed and concurrent systems (components that evolve independently) and applied this to fault detection. However, they did not consider the problem of fault detection within a consensus framework. Our work is motivated by the need to detect faults in electrical power systems. Accordingly, we mention also the work of Kato et al. [23] and Garza et al. [24]. In [23] a multi-agent approach was suggested to locate and isolate fault zones. In [24] this problem was considered within a probabilistic framework using dynamic Bayesian networks, rather than the present consensus framework.

The rest of this chapter is organized as follows. The problem is formulated in Section II. This is followed in Section III with a derivation of the likelihood function for our mixture of Markov chains and the EM procedure for selecting optimal weights. Section IV contains an extension to the case of unobserved Markov chains. Finally, a fault diagnosis scheme and simulation results are presented in Sections V and VI, respectively.

\section{Problem formulation}

It is assumed that each agent has local observations of the state of its subsystem, and that the local model for changes in state is represented by a transition probability matrix. Furthermore, it is assumed that agents can exchange information over a computer network; specifically, each agent can know other agents' transition matrices. The idea is to modify the transition matrices within a group of agents in such a way that, under certain conditions, those of all agents in the group converge to a common transition matrix.

The underlying distributed system is represented by undirected graph $G=(V, E)$ whose vertices $V=\{1, \ldots, n\}$ represent agents (measurement and monitoring points) and whose edges $E(E \subseteq V \times V)$ represent communication links. It is assumed that $G$ itself is connected and composed of one of more complete subgraphs (cliques), each corresponding to a group 
of agents trying to achieve consensus. We assume that the cliques are known in advance (we do not consider a problem of finding them [25]). An example of one such graph is given in Fig. 1, where a 2-vertex clique and a 4-vertex clique have been circled. The neighbourhood of an agent $q$ is defined as the set of agents $\mathcal{N}_{q} \subseteq V$ such that $\mathcal{N}_{q} \triangleq\{p \in V \mid(q, p) \in E\}$. So a given agent can potentially belong to more than one group. This is illustrated in Fig. 1; notice that vertex $q$ belongs to two cliques. We will assume that if an agent is a member of more than one group, it will engage independently in achieving consensus within those groups; it will not share information among the groups. Additionally, we will suppose that communication links between group members are completely reliable with no latency.

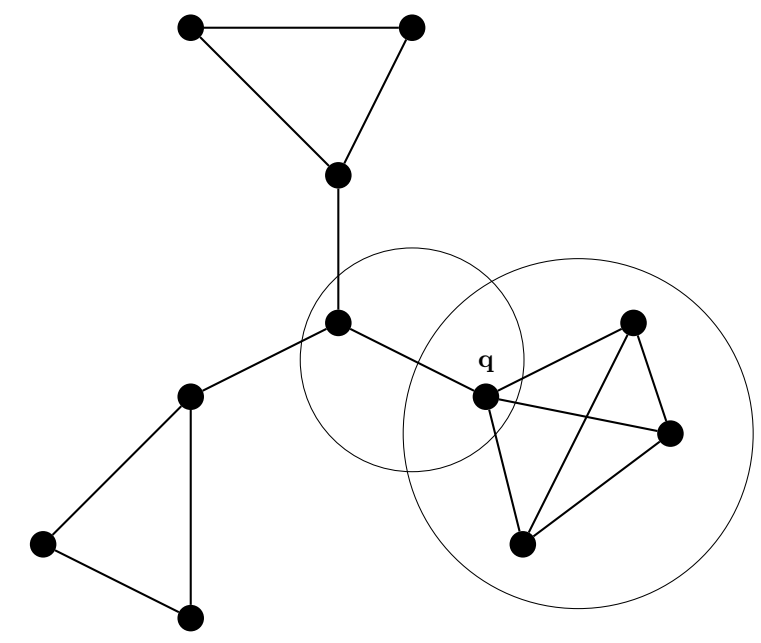

Fig. 1. Communication Graph of an Agent Network

Suppose that there are $K$ agents all of whom have the same set of subsystem states $S=\{1,2, \ldots, N\}$. Starting from iteration $\tau=1$, agent $i$ updates its transition matrix by taking a weighted sum of the other agents' matrices and its own. Let $\mathbf{P}_{i}^{(\tau)}$ be the transition matrix of agent $i$ at iteration $\tau(\tau \geq 1)$. Then,

$$
\mathbf{P}_{i}^{(\tau)}=\sum_{j=1}^{K} \psi_{i j} \mathbf{P}_{j}^{(\tau-1)}, \quad i=1, \ldots, K,
$$

where $\Psi=\left[\psi_{i j}\right]$ is a $K \times K$ is ergodic stochastic matrix with strictly positive entries; $\psi_{i j}$ is a "consensus rating" assigned by agent $i$ to agent $j$ to rate the influence of agent $j$ on agent $i$. The transition matrices $\mathbf{P}_{j}^{(0)}$ at iteration $\tau=0$ are the initially pooled transition matrices. 
The updating procedure at iteration $\tau$ is represented for all agents by

$$
\left[\begin{array}{c}
\mathbf{P}_{1}^{(\tau)} \\
\mathbf{P}_{2}^{(\tau)} \\
\vdots \\
\mathbf{P}_{K}^{(\tau)}
\end{array}\right]=\left[\begin{array}{ccc}
\psi_{11} I & \psi_{12} I & \ldots \psi_{1 K} I \\
\psi_{21} I & \psi_{22} I & \ldots \psi_{2 K} I \\
\vdots & \vdots & \vdots \\
\psi_{K 1} I & \psi_{K 2} I & \ldots \psi_{K K} I
\end{array}\right]\left[\begin{array}{c}
\mathbf{P}_{1}^{(\tau-1)} \\
\mathbf{P}_{2}^{(\tau-1)} \\
\vdots \\
\mathbf{P}_{K}^{(\tau-1)}
\end{array}\right]
$$

where $I$ is the $N \times N$ identity matrix. Defining the group transition matrix at iteration $\tau$ to be the block matrix $\mathcal{P}^{(\tau)}=\left[\mathbf{P}_{1}^{(\tau)}\left|\mathbf{P}_{2}^{(\tau)}\right| \ldots \mid \mathbf{P}_{K}^{(\tau)}\right]^{\top}$, where $T$ denotes transpose, equation (2) is expressed compactly as $\mathcal{P}^{(\tau)}=[\Psi \otimes I] \mathcal{P}^{(\tau-1)}$, or equivalently

$$
\mathcal{P}^{(\tau)}=[\Psi \otimes I]^{(\tau)} \mathcal{P}^{(0)},
$$

where $\otimes$ is the Kronecker product and $\mathcal{P}^{(0)}=\left[\mathbf{P}_{1}^{(0)}\left|\mathbf{P}_{2}^{(0)}\right| \ldots \mid \mathbf{P}_{K}^{(0)}\right]^{\top}$ is the block matrix made up of the initial transition matrices participating in the algorithm. Convergence of (2) is assured under the condition that $\Psi \otimes I$ is a contraction, that is, $\|\Psi \otimes I\| \leq 1$. We exploit the following properties of the Kronecker product ([26], [27]):

Lemma 1: If $A$ is an $m_{A} \times n_{A}$ matrix and $B$ is an $m_{B} \times n_{B}$ matrix, then, for any $p$-norm $\|\cdot\|,\|A \otimes B\|=\|A\|\|B\|$.

Lemma 2: If $A$ and $B$ are square matrices, then $(A \otimes B)^{n}=A^{n} \otimes B^{n}$.

Since $\|\Psi\|_{\infty}=1$ and $\|I\|_{\infty}=1$, applying Lemma 1 to $\Psi \otimes I$ shows that $\|\Psi \otimes I\| \leq 1$. Furthermore, applying Lemma 2 to the group transition matrix $\mathcal{P}^{(\tau)}$, given by (3), we obtain

$$
\mathcal{P}^{(\tau)}=\left[\Psi^{\tau} \otimes I\right] \mathcal{P}^{(0)} .
$$

As $\tau$ goes to infinity $\mathcal{P}^{(\tau)}$ approaches to the group consensus matrix $\mathcal{P}_{c}$ given by

$$
\mathcal{P}_{c}=\left[\begin{array}{c}
\mathbf{P}_{c} \\
\mathbf{P}_{c} \\
\vdots \\
\mathbf{P}_{c}
\end{array}\right]=\left[\begin{array}{ccc}
\pi_{\Psi_{1}} I & \pi_{\Psi_{2}} I \ldots \pi_{\Psi_{K}} I \\
\pi_{\Psi_{1}} I & \pi_{\Psi_{2}} I \ldots & \pi_{\Psi_{K}} I \\
\vdots & \vdots & \vdots \\
\pi_{\Psi_{1}} I & \pi_{\Psi_{2}} I \ldots \pi_{\Psi_{K}} I
\end{array}\right]\left[\begin{array}{c}
\mathbf{P}_{1}^{(0)} \\
\mathbf{P}_{2}^{(0)} \\
\vdots \\
\mathbf{P}_{K}^{(0)}
\end{array}\right]
$$

where $\pi_{\Psi}=\left[\pi_{\Psi_{1}} \pi_{\Psi_{2}} \ldots \pi_{\Psi_{K}}\right]$ is the limiting distribution of the stochastic matrix $\Psi$. Notice that, for the iterative procedure (4) to converge, the weights must be chosen so that $\Psi$ is ergodic [17]. It remains to specify how to estimate entries of $\Psi$ in the first iteration of the algorithm. We note that since the right-hand side of (1) is a mixture of transition matrices 
([28], [29]), the weights $\psi_{i j}$ of agent $i$ can be interpreted as the distribution of a latent variable.

In the next section we derive the likelihood function for our Markov chain mixture, which is used in the subsequent EM framework to estimate our consensus ratings. Once the optimal ratings are estimated and $\Psi$ is formed, its stationary distribution (denoted by $\pi_{\Psi}$ ) can be evaluated [30]. From that and the initially pooled transition matrices $\mathcal{P}^{(0)}$, an estimated consensus transition matrix $\mathbf{P}_{c}$ and corresponding stationary distribution $\pi_{c}$ can be determined.

\section{Estimation OF OPTIMAL CONSENSUS RATINGS}

To estimate concensus we must first determine a likelihood function for the linear combination of transition matrices involved in the consensus scheme for the $i^{\text {th }}$ agent in (2) for $\tau=1$. For simplicity let us write

$$
\mathbf{P}_{i}=\sum_{j=1}^{K} \psi_{j} \mathbf{P}_{j} .
$$

The iteration indices have been omitted, and $\psi_{i j}$ has been replaced by $\psi_{j}$. In a group of agents it is assumed that each has observed its own state sequence and corresponding transition matrix. When a particular agent $i$ revises its own transition matrix, it invites the other agents to transmit theirs. Agent $i$ then adapts its own transition matrix based on the information received. We will explain how the consensus weights depend on the state sequences and the corresponding transition probabilities of each of the agents in the group. We follow an approach of Anderson and Goodman [31], but extended this to Markov chain mixtures.

It is assumed that the state sequence $\left\{X_{t}, 0 \leq t \leq T\right\}$ of agent $i$ is governed by $\mathbf{P}_{i}$ in (6). Transitions in this sequence are obtained as a mixture of sequences $X_{t}^{k}, k=1, \ldots, K$, of all $K$ agents in the group. Since each agent $k$ is weighted by some value $\psi_{j}$, the probability of a particular transition at time $t$, from state $x_{i-1}$ to state $x_{i}$, can be modelled as the product of two probabilities: the probability of a transition from one state to another and the probability that the transition itself is caused by agent $k$. Consequently, the probability of the state sequence $x_{0}, \ldots, x_{T}$ is

$$
\psi_{\left(x_{0} x_{1}\right)_{k}} p_{\left(x_{0} x_{1}\right)_{k}} \psi_{\left(x_{1} x_{2}\right)_{k}} p_{\left(x_{1} x_{2}\right)_{k}} \ldots \psi_{\left(x_{T-1} x_{T}\right)_{k}} p_{\left(x_{T-1} x_{T}\right)_{k}}
$$


Expression (7) can be further extended by introducing a random process $\left(Z_{t}\right)$ to model random selection of the source $k$ of a particular transition from $x_{i-1}$ to $x_{i}$. Since this transition at time $t$ can come from only one source, an indicator $I_{\left\{Z_{t}=k\right\}}$ of this source is introduced. In that case, the weight $\psi_{\left(x_{i-1} x_{i}\right)_{k}}$ can be interpreted as the probability that a particular transition probability $p_{\left(x_{i-1} x_{i}\right)_{k}}$ comes from agent $k$, denoted as $P\left(Z_{t}=k\right)$. Thus, for each transition from $x_{i-1}$ to $x_{i}$, expression $(7)$ is modified to obtain

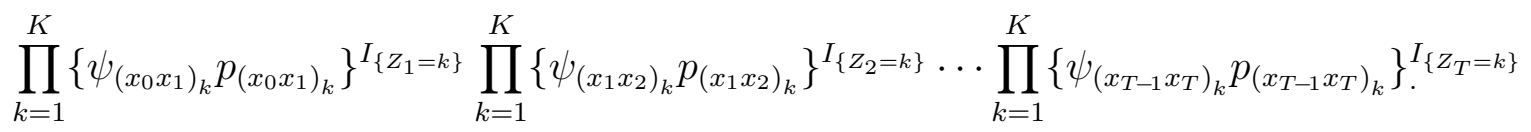

The next step towards calculating (7) requires counting the number of transitions, from $x_{i-1}$ to $x_{i}$ for agent $k$ until time $t$ on the entire sequence, as follows:

$$
\begin{gathered}
\prod_{x_{0} x_{1}} \prod_{k=1}^{K}\left\{\psi_{\left(x_{0} x_{1}\right)_{k}} p_{\left(x_{0} x_{1}\right)_{k}}\right\}^{I_{\left\{Z_{1}=k\right\}} N_{1}\left(x_{0} x_{1}\right)_{k}} \prod_{x_{1} x_{2}} \prod_{k=1}^{K}\left\{\psi_{\left(x_{1} x_{2}\right)_{k}} p_{\left(x_{1} x_{2}\right)_{k}}\right\}^{I_{\left\{Z_{2}=k\right\}} N_{2}\left(x_{1} x_{2}\right)_{k}} \\
\cdots \prod_{x_{T-1} x_{T}} \prod_{k=1}^{K}\left\{\psi_{\left(x_{T-1} x_{T}\right)_{k}} p_{\left(x_{T-1} x_{T}\right)_{k}}\right\}^{I_{\left\{Z_{T}=k\right\}} N_{T}\left(x_{T-1} x_{T}\right)_{k}},
\end{gathered}
$$

or, more compactly,

$$
\prod_{t=1}^{T} \prod_{i, j=1}^{N} \prod_{k=1}^{K}\left\{P\left(Z_{t}=k\right) P\left(X_{t}=j \mid X_{t-1}=i, Z_{t}=k\right)\right\}_{\left\{Z_{t}=k\right\}}^{I_{t} N_{t}\left(X_{t-1}=i, X_{t}=j\right)_{k}} .
$$

To simplify notation, $P\left(Z_{t}=k\right)$ will be denoted as $\psi_{k}$, the transition probability $P\left(X_{t}=\right.$ $j \mid X_{t-1}=i, Z_{t}=k$ ) will be given in the shortened form $P_{t}(i, j)_{k}$ and the number of transitions in a state sequence $X_{t}^{k}$ by time $t$ for a particular agent $k$ by $N_{t}(i, j)_{k}$ : (10) becomes

$$
\prod_{t=1}^{T} \prod_{i, j=1}^{N} \prod_{k=1}^{K}\left\{\psi_{k} P_{t}(i, j)_{k}\right\}^{I_{\left\{Z_{t}=k\right\}} N_{t}(i, j)_{k}}
$$

It is apparent from (11) that the random variable $Z_{t}$ is not directly observable. However, this incomplete-data problem can be converted to a complete-data problem; if the problem is extended to find the likelihood of the sequence $\left\{\left(X_{t}, Z_{t}\right), 0 \leq t \leq T\right\}$ instead, it opens up the possibility of using the EM framework [32].

As previously discussed, expression (11) is a likelihood function of the complete-data vector whose logarithm is given by

$$
\log L(\Psi ; \boldsymbol{X}, \boldsymbol{Z})=\sum_{t=1}^{T} \sum_{i, j=1}^{N} \sum_{k=1}^{K} I_{\left\{Z_{t}=k\right\}} N_{t}(i, j)_{k}\left\{\log P_{t}(i, j)_{k}+\log \psi_{k}\right\}
$$

The EM algorithm is a two-step iterative procedure. In the first step, called the E-step, the $Q$ function is calculated, which is the mathematical expectation of (12) given observations 
$\left\{X_{t}, 0 \leq t \leq T\right\}: Q\left(\psi_{k} \mid \psi_{k}^{(\iota)}\right)=\mathbb{E}_{\boldsymbol{Z} \mid \boldsymbol{X}, \boldsymbol{\Psi}}\{\log L(\boldsymbol{\Psi} ; \boldsymbol{X}, \boldsymbol{Z})\}$, where $\psi_{k}^{(\iota)}$ is a set of parameter estimated in previous iteration $\iota$. The $Q$ function evaluation is reduced to computing the mathematical expectations of indicator functions, because the transition probabilities $P_{t}(i, j)_{k}$, counts $N_{t}(i, j)_{k}$ and initial mixing proportions $\psi_{k}^{(\ell)}$ are known in advance. By Bayes' Theorem

$$
P(A \mid B \cap C)=\frac{P(B \mid A \cap C) P(A \mid C)}{P(B \mid C)} .
$$

Furthermore, assuming that $X_{t}$ depends only on $X_{t-1}$ and $I_{\left\{Z_{t}=k\right\}}$, as well as presuming that $I_{\left\{Z_{t}=k\right\}}$ and $X_{t-1}$ are independent, the mathematical expectation of the indicator function is given as follows:

$$
\begin{aligned}
\mathbb{E}_{\boldsymbol{Z} \mid \boldsymbol{X}, \boldsymbol{\Psi}}\left\{I_{\left\{Z_{t}=k\right\}} \mid \mathbf{X} ; \boldsymbol{\Psi}\right\} & =\frac{P\left(X_{t}=j \mid X_{t-1}=i, I_{\left\{Z_{t}=k\right\}}=1\right) P\left(I_{\left\{Z_{t}=k\right\}}=1\right)}{P\left(X_{t}=j \mid X_{t-1}=j\right)} \\
& =\frac{P\left(X_{t}=j \mid X_{t-1}=i, I_{\left\{Z_{t}=k\right\}}=1\right) P\left(I_{\left\{Z_{t}=k\right\}}=1\right)}{\sum_{h=1}^{K} P\left(X_{t}=j \mid X_{t-1}=i, I_{\left\{Z_{t}=h\right\}}=1\right) P\left(I_{\left\{Z_{t}=h\right\}}=1\right)} \\
& =\frac{\psi_{k}^{(\iota)} P_{t}(i, j)_{k}}{\sum_{h=1}^{K} \psi_{h}^{(\iota)} P_{t}(i, j)_{h}}=\varphi_{t}^{(\iota)}(i, j)_{k} .
\end{aligned}
$$

Finally an expression for the $Q$ function is given by

$$
Q\left(\psi_{k} \mid \psi_{k}^{(\iota)}\right)=\sum_{t=1}^{T} \sum_{i, j=1}^{N} \sum_{k=1}^{K} \varphi_{t}^{(\iota)}(i, j)_{k} N_{t}(i, j)_{k}\left\{\log P_{t}(i, j)_{k}+\log \psi_{k}\right\} .
$$

In the second step of the EM algorithm, called the M-step, previously assumed parameters are optimized based on the expectation of the log likelihood: $\psi_{k}^{(\iota+1)}=\arg \max _{\psi_{k}} Q\left(\psi_{k} \mid \psi_{k}^{(\iota)}\right)$. Introducing a Lagrange multiplier $\mu$ into (15) for the constraint $\sum_{k=1}^{K} \psi_{k}=1$, we obtain

$$
Q\left(\psi_{k} \mid \psi_{k}^{(\iota)}\right)=\sum_{t=1}^{T} \sum_{i, j=1}^{N} \sum_{k=1}^{K} \varphi_{t}^{(\iota)}(i, j)_{k} N_{t}(i, j)_{k}\left\{\log P_{t}(i, j)_{k}+\log \psi_{k}\right\}-\mu\left(\sum_{k=1}^{K} \psi_{k}-1\right) .
$$

After taking the derivative of $Q$ with respect to $\psi_{k}$ we get

$$
\frac{\partial Q\left(\psi_{k} \mid \psi_{k}^{(\iota)}\right)}{\partial \psi_{k}}=\frac{\sum_{t=1}^{T} \sum_{i, j=1}^{N} \varphi_{t}^{(\iota)}(i, j)_{k} N_{t}(i, j)_{k}}{\psi_{k}}-\mu=0 .
$$

Rearranging (17) and using the constraint we obtain

$$
\mu=\sum_{h=1}^{K} \sum_{t=1}^{T} \sum_{i, j=1}^{N} \varphi_{t}^{(\iota)}(i, j)_{h} N_{t}(i, j)_{h} .
$$

Finally, the updated equation for $\psi_{k}$ is given by

$$
\psi_{k}=\frac{\sum_{t=1}^{T} \sum_{i, j=1}^{N} \varphi_{t}^{(\ell)}(i, j)_{k} N_{t}(i, j)_{k}}{\sum_{h=1}^{K} \sum_{t=1}^{T} \sum_{i, j=1}^{N} \varphi_{t}^{(\iota)}(i, j)_{h} N_{t}(i, j)_{h}} .
$$


By altering the E-step and M-step, in each iteration $\iota$, the function $Q\left(\psi_{k} \mid \psi_{k}^{(\iota)}\right)$ is calculated and the parameters are optimized. From an implementation point of view there are two ways to halt the procedure. The first is when the difference in the value of $Q$ is below some threshold $\Theta$ assumed in advance, that is, $Q\left(\psi_{k} \mid \psi_{k}^{(\iota)}\right)-Q\left(\psi_{k} \mid \psi_{k}^{(\iota-1)}\right) \leq \Theta$. The second is to specify in advance the total number of iterations $\Upsilon$ and stop when $\iota \geq \Upsilon$.

Taking into consideration the E-step and M-step used to estimate optimal ratings of the stochastic matrix $\Psi$, it is apparent that there are two specific kinds of information each agent in the group requires. Firstly, by (14) it follows that each agent in the group has to know the other agents' transition probabilities. In other words, information on the models perceived by the group members are supposed to be shared among the group. Secondly, even more interesting conclusions can be drawn from (18). In order to rate other group members, agent $i$ relies on information regarding a number of transitions, $N_{t}^{k}, k=1, \ldots, K$, of pooled state sequences $X_{t}^{k}$. As we will see shortly, a major problem in applying our algorithm is related to the inability to observe these state sequences directly.

Before proceeding, recall briefly the notation introduced of this section. The index $i$, denoting the agent that revises its distribution, is omitted: $X_{t}^{k}$ is shorthand for the state sequence $X_{t}^{i k}$ that models the influence of agent $k$ on agent $i$, and $N_{t}^{k}$ is short for $N_{t}^{i k}$, the number of transitions. Which notation we will use depends on the context.

\section{Extension to Unobservable CASE}

It is clearly unrealistic to assume that each state transition is an observable discrete event, because of measurement noise or because the observed signal is generated by one of multiple sources randomly switched by an unobservable Markov chain, as depicted in Fig. 2. It is therefore desirable to extend our algorithm to the case where the states are hidden.

In order to adapt our algorithm to the context of Hidden Markov Models (HMMs) ([33], [34]), it will be necessary to establish a link with the solutions of the three essential problems connected with HMMs described by Rabiner [33]. The basic elements of a HMM are the number of states $N$; the number of distinct observation symbols (discrete observations) $O=\left\{o_{1}, o_{2}, \ldots, o_{M}\right\}$, or the number of mixture components (continuous observations) $M$; and the state transition probabilities $\boldsymbol{A}=\left[\alpha_{i j}\right]_{N \times N}$, where

$$
\alpha_{i j} \equiv P\left(X_{t}=j \mid X_{t-1}=i\right), \quad 1 \leq i, j \leq N .
$$


The next element is an observation probability defined as a matrix $\boldsymbol{B}=\left[\beta_{j}\left(y_{t}\right)\right]_{N \times 1}$, where $\beta_{j}\left(y_{t}\right)$ is a probability of a particular observation. For discrete observations,

$$
\beta_{j}\left(y_{t}\right) \equiv P\left(Y_{t}=o_{m} \mid X_{t}=j\right), \quad 1 \leq j \leq N, 1 \leq m \leq M
$$

The final element is the initial state distribution $\boldsymbol{\Pi}=\left[\pi_{k}\right]_{N \times 1}$. We use the following compact notation:

$$
\lambda=(\boldsymbol{A}, \boldsymbol{B}, \boldsymbol{\Pi}),
$$

where $N$ and $M$ are defined implicitly.

The three basic problems identified by Rabiner [33] are as follows.

Problem 1 : The efficient computation of the probability $P(Y \mid \lambda)$ of the observed sequence $Y=Y_{1}, Y_{2}, \ldots, Y_{T}$, given the model $\lambda$.

Problem 2 : Estimation of the hidden state sequence $X=X_{1}, X_{2}, \ldots, X_{T}$, given the observation sequence $Y=Y_{1}, Y_{2}, \ldots, Y_{T}$ and the model $\lambda$.

Problem 3 : Estimation of the parameters of model $\lambda$ that maximize $P(Y \mid \lambda)$.

To solve Problem 1 the forward-backward procedure [33] is applied, which is a recursive method for efficient calculation of the probability of the observed sequence given the model $\lambda$. To maximize the probability of a hidden state sequence for given observations, the Viterbi algorithm [35] is used to address Problem 2. Finally, Problem 3 is addressed applying the Baum-Welch algorithm [36].

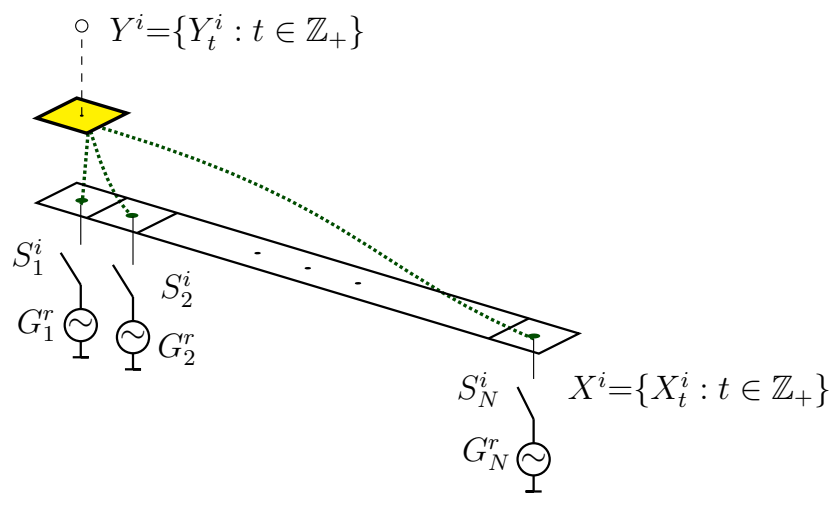

Fig. 2. Local Hidden Markov Model

The connection with our algorithm will now be explained. As given previously, each agent $i$ in the group takes observations $Y_{t}^{i}=\left\{y_{1}^{i}, y_{2}^{i}, \ldots, y_{T}^{i}\right\}$, independently allowing the application 
of a consensus algorithm. In addition, it is assumed that each of these observations is generated by a Markov switching model. In other words, a particular observation at time $t$ comes from one of the underlying signal generators $G_{i}^{r}, r=1, \ldots, R, i=1, \ldots, N$, where $R$ is a number of working regimes and $N$ is the number of signal generators. We do not assume anything about these hypothetical generators. Indeed we are only interested in the sequence of their activations/deactivations, modelled by an associated Markov chain $X^{i}=\left\{X_{t}^{i}, 0 \leq t \leq T\right\}$ defined on a state space $S=\{1,2, \ldots, N\}$ with a corresponding transition probability matrix $\boldsymbol{A}_{r}$. It is very important to note that that an unobservable Markov chain transition matrix $\boldsymbol{A}_{r}$ will be used to form the initial pool of Markov chain transition matrices in (5), that is, if agent $i$ is in the working regime $r$ then $\boldsymbol{A}_{r} \equiv P_{i}^{(0)}$. Note that the number of generators $N$ defines the size of $S$ and that number is a same for all working regimes. Consequently, all transition probability matrices are the same size.

In the context of the algorithm described in Sections II and III, it is necessary for each operating mode $r$ to determine a transition probability matrix $\boldsymbol{A}_{r}$ of an unobservable Markov chain $X^{i}$ of corresponding hypothetical switches $S^{i}=\left\{S_{1}^{i}, \ldots, S_{N}^{i}\right\}, i=1, \ldots,|V|$, and similarly for $\boldsymbol{B}_{r}$ and the initial state distribution $\boldsymbol{\Pi}_{r}$. With this in mind, it is apparent that the Baum-Welch algorithm provides a means of estimating different working regimes and designing a bank of competing models $\Lambda=\left\{\lambda\left(\Theta_{1}\right) \ldots \lambda\left(\Theta_{R}\right)\right\}$. These models describe possible working conditions of each subsystem, where $\lambda\left(\Theta_{r}\right)$ is model $(21)$ and $\Theta_{r}=\left(\boldsymbol{A}_{r}, \boldsymbol{B}_{r}, \boldsymbol{\Pi}_{r}\right)$ is the vector of the parameters of model $r$. The currently active model of a particular agent $i$ is determined by the forward-backward procedure, selecting the most likely model $\lambda_{i}=\lambda\left(\Theta_{r}\right)$.

Finally, as per the proposed consensus scheme, to estimate a rating $\psi_{i k}$ using (18), agent $i$ needs to estimate the number of transitions, $N_{t}^{k}$, in the underlying state sequence $X_{t}^{k}$. First, $X_{t}^{i k}$ must include in it information about a model of the agent $k$, whose rating is being assessed. Second, local observations $Y_{t}^{i}$ of the agent $i$ are used to revise its distribution. Thus, the Viterbi algorithm logically connects these two aspects, all with the aim of estimating the state sequences $X_{t}^{i k}$. Of course after $X_{t}^{i k}$ is estimated, $N_{t}^{k}$ is easily determined. So, to estimate the optimal ratings $\psi_{i k}$, the Viterbi algorithm is applied to estimate the hidden sequences $X_{t}^{i k}=\left\{x_{1}^{i k}, x_{2}^{i k}, \ldots, x_{T}^{i k}\right\}$ from local models $\lambda_{k}$ of the all agents in the group and local observations $Y_{t}^{i}=\left\{y_{1}^{i}, y_{2}^{i}, \ldots, y_{T}^{i}\right\}$.

To help explain an application of the algorithm to real-time systems, a sequence diagram 
is given in Fig. 3, the steps summarized as follows:

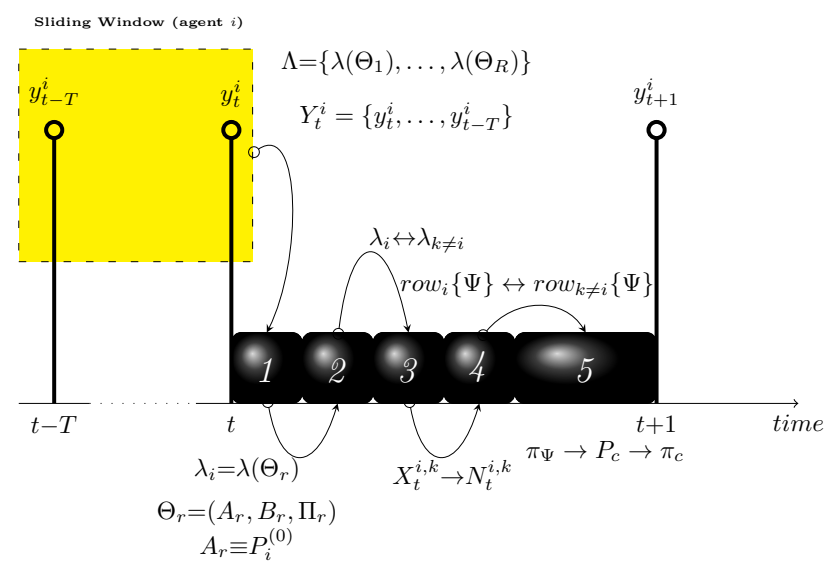

Fig. 3. An Algorithm State Transition Diagram

Step 0: At time $t$ each agent $i$ from the group of $K$ agents has collected $T$ observations $Y_{t}^{i}=\left\{y_{1}^{i}, y_{2}^{i}, \ldots, y_{T}^{i}\right\}$ within the sliding window. Additionally every agent in the group possesses a bank of competing models $\Lambda=\left\{\lambda\left(\Theta_{1}\right) \ldots \lambda\left(\Theta_{R}\right)\right\}$;

Step 1: By applying the forward-backward recursive procedure to a bank $\Lambda$, given a set of observations $Y_{t}^{i}$, a currently active model $\lambda_{i}=\lambda\left(\Theta_{r}\right)$ of agent $i$, with a set of parameters $\Theta_{r}=\left(A_{r}, B_{r}, \Pi_{r}\right)$, is determined. Note that initial transition matrices $P_{i}^{(0)}$ of consensus algorithm and model transition matrices $A_{r}$ the same $\left(P_{i}^{(0)} \equiv A_{r}\right)$;

Step 2: All agents in the group exchange, over a computer network, currently active models $\lambda_{i} \leftrightarrow \lambda_{k \neq i}, k=1, \ldots, K$

Step 3: By the means of the Viterbi algorithm every agent $i$ estimates the unobservable sequences $X^{i k}$ and corresponding transitions $N_{t}^{i k}$ from $Y_{t}^{i}$ and $\lambda_{k}, k=1, \ldots, K$. Using the EM algorithm an agent $i$ estimates a row $i$ of a stochastic matrix $\Psi$, $\operatorname{row}_{i}\{\Psi\}=\left(\psi_{i 1}, \ldots, \psi_{i K}\right)$;

Step 4: The group of agents exchange over the computer network rows of the stochastic matrix $\Psi \operatorname{row}_{i}\{\Psi\} \leftrightarrow \operatorname{row}_{k \neq i}\{\Psi\}$, allowing each agent to form the matrix $\Psi$

Step 5: Once these transactions are complete, each agent computes a stationary distribution [30] $\pi_{\Psi}=\left(\pi_{\psi_{1}}, \ldots, \pi_{\psi_{K}}\right)$ required to estimate a consensus transition matrix $\mathbf{P}_{c}$, from which the stationary distribution $\pi_{c}$ is computed. After a stationary 
distribution $\pi_{c}$ is estimated, a fault detection scheme is applied, as explained in the next section.

\section{Fault detection scheme}

As explained in Section I, an important aspect of our fault detection scheme is a distance measurement between the stationary distribution $\pi_{c}$ of the consensus transition matrix and the stationary distribution $\pi_{P^{(0)}}$ of the agent's initial transition matrix. To measure the distance between the two stationary distributions, $\pi_{1}$ and $\pi_{2}$, we will use the $L_{2}$-norm:

$$
\delta\left(\pi_{1}, \pi_{2}\right)=\sum_{i=1}^{N}\left(\sum_{j=1}^{i} \pi_{1}(j)-\sum_{j=1}^{i} \pi_{2}(j)\right)^{2},
$$

where $N$ is a number of discrete states. Once evaluated, these distances are stored to form a fault table (or dictionary). Because the table is symmetric, the total number of different values, as a function of the number of operation modes $n$, is $\frac{1}{2} n(n-1)$. As noted earlier,

TABLE I

The Fault Table (Dictionary)

\begin{tabular}{c|cccc}
$\delta\left(\pi_{A_{i}}, \pi_{A_{j}}\right)$ & $\pi_{\mathbf{A}_{1}}$ & $\pi_{\mathbf{A}_{2}}$ & $\ldots$ & $\pi_{\mathbf{A}_{R}}$ \\
\hline$\pi_{\mathbf{A}_{1}}$ & 0 & $\delta_{12}$ & $\ldots$ & $\delta_{1 R}$ \\
$\pi_{\mathbf{A}_{2}}$ & $\delta_{12}$ & 0 & $\ldots$ & $\delta_{2 R}$ \\
$\vdots$ & $\vdots$ & $\vdots$ & $\vdots$ & $\vdots$ \\
$\pi_{\mathbf{A}_{R}}$ & $\delta_{1 R}$ & $\delta_{2 R}$ & $\cdots$ & 0
\end{tabular}

every fault diagnosis scheme consists of a series of tasks, the first of which, fault detection, is implemented as Step 5 (above). Here we measure the distance between the stationary distribution of the consensus transition matrix and the stationary distribution of the agent's local transition matrix (model). This step allows us to identify deviations from normal operation. 


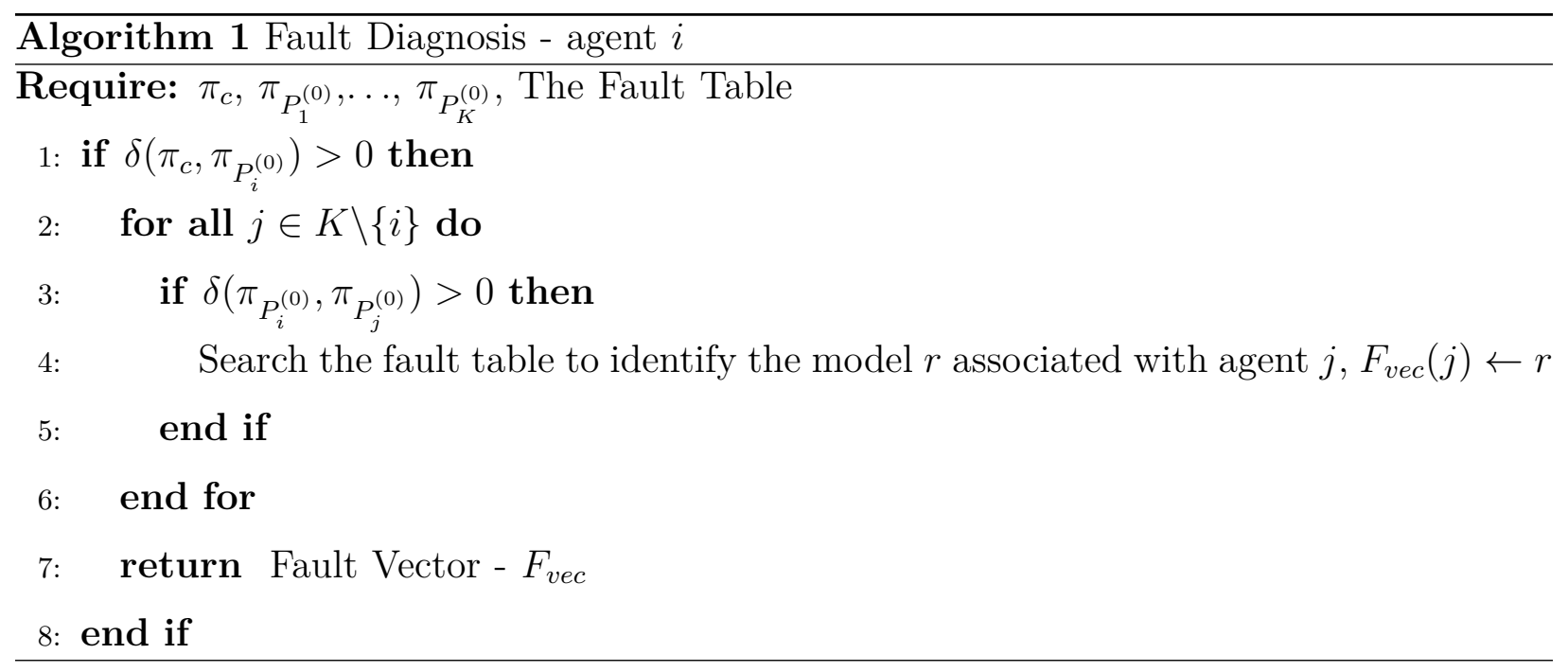

Once a fault is detected, the second task, fault identification, begins. Multiple faults in monitored subsystems can be identified, because all agents have the fault table that summarizes information about different working regimes and each has information about the currently active modes of all other agents in the group. It has become apparent that a potential pitfall of this approach, which is particularly evident when the number of models is large, lies in the need to retrieve the fault table. Consideration of this issue will be part of future research.

A realization of the first and second tasks is summarized in Algorithm 1, which is executed for all agents $i$. In the third fundamental task, fault analysis, we analyse the timevarying characteristics of the fault(s), which are connected to model observations.

\section{Simulation RESUlts}

We illustrate the method using a hypothetical system composed of three interconnected subsystems $(K=3)$. The corresponding agents are labelled 1, 2 and 3 . We supposed that there are three possible subsystem working regimes, each modelled by a HMM with two states $(N=2)$, an unobservable Markov chain and a discrete observations set $O=\{1,2, \ldots, 7\}$ $(M=7)$.

The HMM labelled $\lambda_{1}$ models normal operation, while those labelled $\lambda_{2}$ and $\lambda_{3}$ represent faults. The parameters of these models are given in Table II, III and IV, respectively.

Together, they form a bank of competing models $\Lambda=\left\{\lambda\left(\Theta_{1}\right), \lambda\left(\Theta_{1}\right), \lambda\left(\Theta_{3}\right)\right\}$. The dynamics are rather simple and emulate dynamical changes in signal amplitude. Notwithstanding 
TABLE II

NORMAL WORKING REGIME $\lambda_{1}$

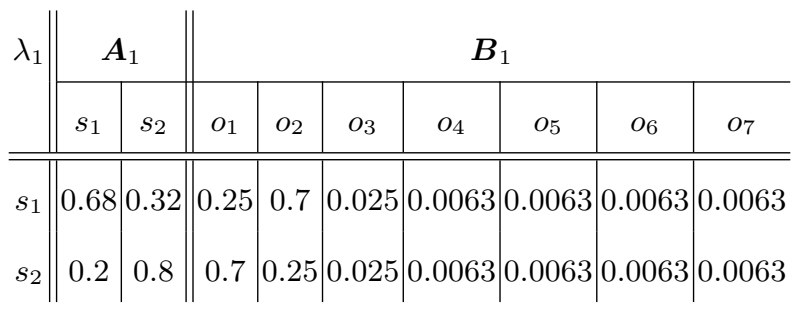

TABLE III

FAUlTy WORKING REGIME $\lambda_{2}$

\begin{tabular}{|c|c|c|c|c|c|c|c|c|c|}
\hline \multirow[t]{2}{*}{$\lambda_{2}$} & \multicolumn{2}{|c|}{$A_{2}$} & \multicolumn{7}{|c|}{$\boldsymbol{B}_{2}$} \\
\hline & $s_{1}$ & $s_{2}$ & $o_{1}$ & $\mathrm{O}_{2}$ & $O_{3}$ & $O_{4}$ & $O_{5}$ & $O_{6}$ & $O_{7}$ \\
\hline$s_{1}$ & 0.4 & 0.6 & 0.0125 & 0.05 & 0.1 & 0.65 & 0.15 & 0.025 & 0.0125 \\
\hline$s_{2}$ & 0.75 & 0.25 & 0.0063 & 0.025 & 0.65 & 0.15 & 0.15 & 0.0125 & 0.0063 \\
\hline
\end{tabular}

this, these simplified models serve to illustrate collective decision-making in a group of agents. Fig. 4 depicts agents' local observations. Agents collect observations by means of a sliding window that contains, at time $t$, the last $T$ samples of the monitored signal. For each agent $i$ in the group, a fault diagnosis scheme is applied to local observations $Y_{t}^{i}=\left\{y_{1}^{i}, y_{2}^{i}, \ldots, y_{T}^{i}\right\}, i=1,2,3$ (in our case, the window length is $T=7$ ).

We analyse the test case depicted in Fig. 4. The transition of agent 1 from mode $\lambda\left(\Theta_{1}\right)$ to $\lambda\left(\Theta_{2}\right)$ occurs at $t=101$. At $t=201$, agent 1 returns to mode $\lambda\left(\Theta_{1}\right)$, while agents 2 and 3 transition to modes $\lambda\left(\Theta_{3}\right)$ and $\lambda\left(\Theta_{2}\right)$, respectively. At $t=301$, all agents are once again in mode $\lambda\left(\Theta_{1}\right)$.

In Step 1 of the state transition diagram (Fig. 3), the forward-backward recursive procedure applies for estimating a currently active model using the bank of models $\Lambda$ and the agent's local observations $Y_{t}^{i}$. The changes of model indexes are depicted in Fig. 5. The dashed lines represent true index values, while the solid lines are estimates obtained from the forward-backward procedure. Notice that there is some delay in identifying these changes. Agents' decision delay $\Delta$, as a function of a sliding window length, is given in Table V. 
TABLE IV

FAULTY WORKING REGIME $\lambda_{3}$

\begin{tabular}{c||c|c||c|c|c|c|c|c|c}
\multicolumn{1}{c||}{$\lambda_{3}$} & \multicolumn{2}{c||}{$\boldsymbol{A}_{3}$} & \multicolumn{6}{c}{$B_{3}$} \\
\cline { 2 - 9 } & $s_{1}$ & $s_{2}$ & $o_{1}$ & $o_{2}$ & $o_{3}$ & $o_{4}$ & $o_{5}$ & $o_{6}$ & $o_{7}$ \\
\hline \hline$s_{1}$ & 0.3 & 0.7 & 0.0125 & 0.0125 & 0.0125 & 0.0125 & 0.0125 & 0.25 & 0.7 \\
$s_{2}$ & 0.71 & 0.29 & 0.0125 & 0.0125 & 0.0125 & 0.0125 & 0.05 & 0.7 & 0.2
\end{tabular}
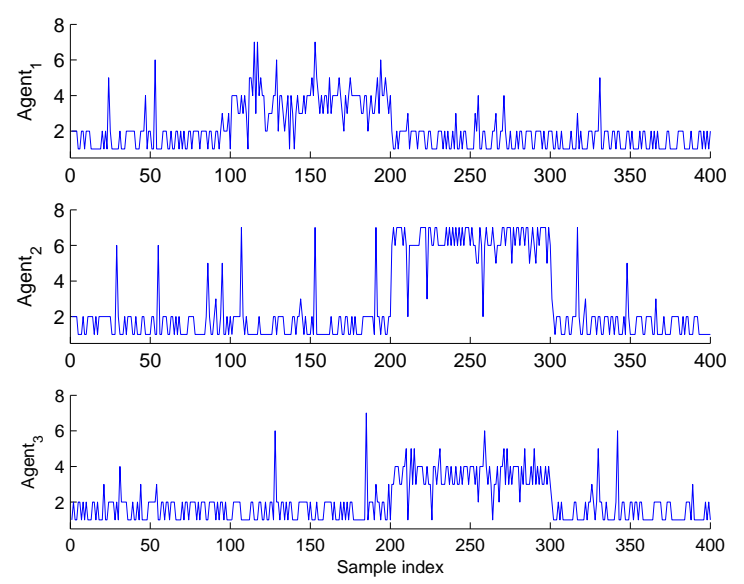

Fig. 4. Agents' Observations

Next, in Step 2, the agents mutually exchange information on current working regime models $\lambda_{k}=\lambda\left(\Theta_{r}\right)$, meaning that agent $k$ has identified a model $r$.

In Step 3 the Viterbi algorithm is used to estimate the state sequences $X_{t}^{i k}, i, k=1,2,3$, given observations $Y_{t}^{i}$ and model parameters $\lambda_{k}=\lambda\left(\Theta_{r}\right)$ in the group. Once all agents in the group estimate their own set of sequences $X_{t}^{i k}$ and count number of transitions $N_{t}^{i k}$ they will estimate rows, $\operatorname{row}_{i}\{\Psi\} i=1,2,3$, of the consensus matrix $\Psi$ using the EM procedure. Numerical examples of optimal ratings estimation of $\Psi$ will be given for three different cases: $t=50,150$ and 250. Simulation results are presented in Figs. 6, 7 and 8, which assume that parameters of the EM algorithm are initialized randomly. These figures demonstrate how agents' rates evolve with the EM iterations. As described before, each agent in the group rates itself and other agents. Agent 1's rates, $\psi_{11}, \psi_{21}$ and $\psi_{31}$, are a measure of the 

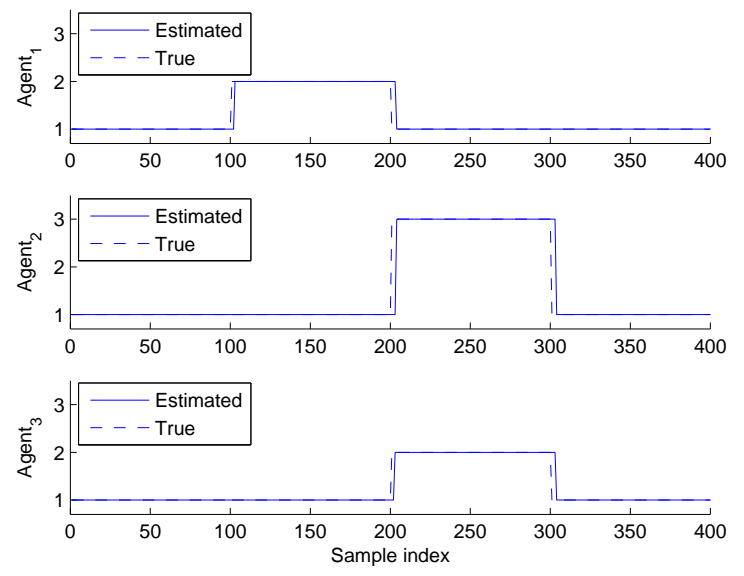

Fig. 5. Agents' Model Indexes

TABLE V

Decision Delay $\Delta$ as a Function of a Sliding Window Length $T$

$$
\begin{array}{l|llll}
T & 5 & 7 & 9 & 11 \\
\hline \Delta & 2 & 3 & 4 & 6
\end{array}
$$

influence of agent 1 working regime model on the group. These values form the first column of the stochastic matrix $\Psi \equiv \Psi^{(0)}$. As we showed in Section II, the first column of $\Psi^{(\tau)}$ in the limit as $\tau \rightarrow \infty$ will be the stationary value $\pi_{\psi_{1}}$. Similarly, for agents 2 and 3 , their estimated rates, $\psi_{12}, \psi_{22}, \psi_{32}$, and $\psi_{13}, \psi_{23}, \psi_{33}$, converge to stationary values $\pi_{\psi_{2}}$ and $\pi_{\psi_{3}}$, respectively. Stochastic matrices $\Psi$, for $t=150$ and 250, and their stationary distributions, are summarized in Tables VI and VII.

TABLE VI

Initial CONSEnsus Matrix FOR $t=150$

$$
\begin{array}{l|lll} 
& 0.2772 & 0.3614 & 0.3614 \\
\Psi_{t=150}^{(0)} & 0.3482 & 0.3259 & 0.3259 \\
& 0.3618 & 0.3191 & 0.3191 \\
\hline \pi_{\Psi_{t=150}} & 0.3294 & 0.3353 & 0.3353
\end{array}
$$



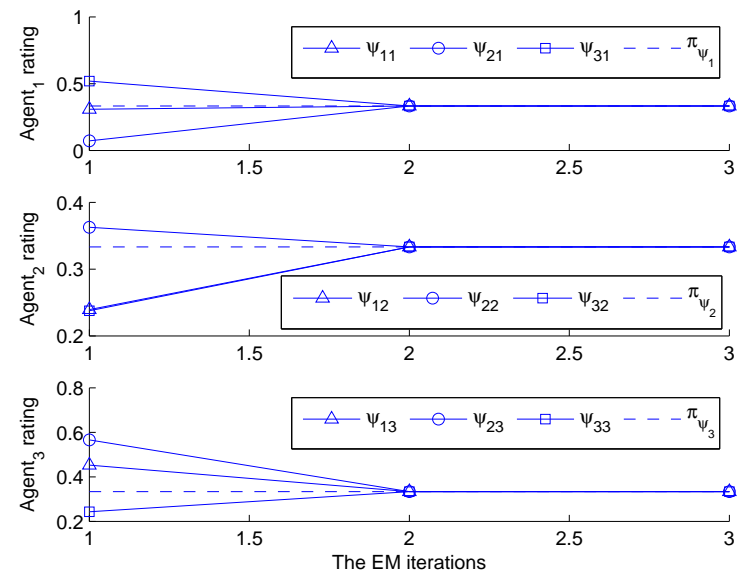

Fig. 6. Consensus Matrix Estimation at $t=50$
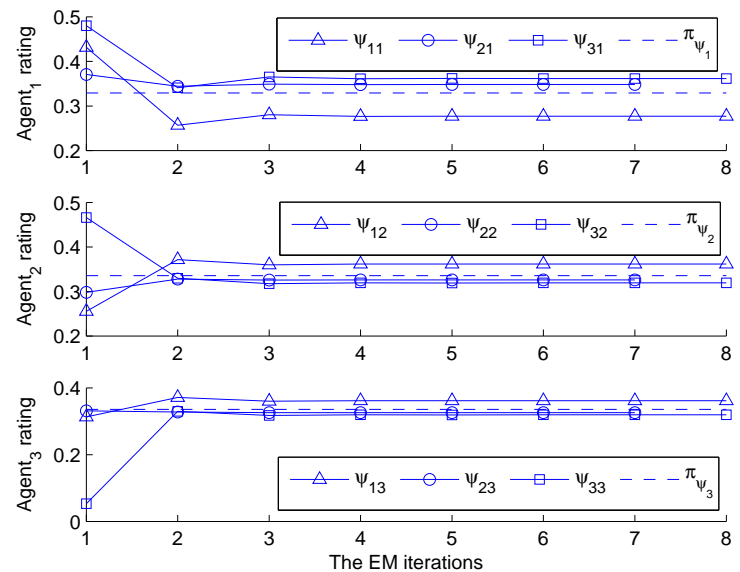

Fig. 7. Consensus Matrix Estimation at $t=150$

Another important conclusion can be drawn from Figs. 6, 7 and 8. It concerns the stopping criteria of the EM algorithm, which combines a fixed number of iterations, $\Upsilon=10$, with a threshold value, $\Theta=10^{-4}$. Selection of these parameters is an essential part of the practical implementation of our algorithm to provide real-time response.

In Step 4, previously estimated rows of the matrix $\Psi, \operatorname{row}_{i}\{\Psi\}=\left(\psi_{i 1}, \ldots, \psi_{i K}\right) i=$ $1, \ldots, K$, are exchanged among group members to form a stochastic matrix $\Psi$. 

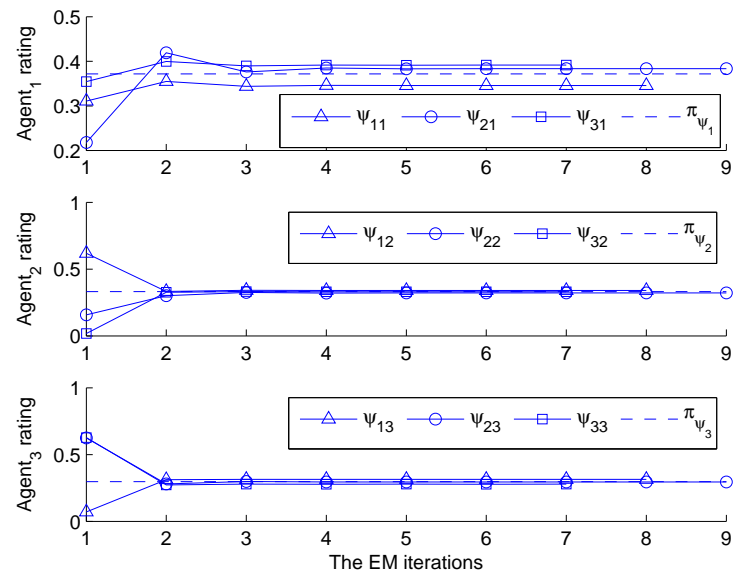

Fig. 8. Consensus Matrix Estimation at $t=250$

TABLE VII

Initial Consensus Matrix for $t=250$

\begin{tabular}{l|lll} 
& 0.3457 & 0.3403 & 0.3140 \\
$\Psi_{t=250}^{(0)}$ & 0.3837 & 0.3213 & 0.2950 \\
& 0.3916 & 0.3302 & 0.2782 \\
\hline$\pi_{\Psi_{t=250}}$ & 0.3719 & 0.3310 & 0.2971
\end{tabular}

Finally, in Step 5, once each agent in the group has its own stochastic matrix $\Psi$, with stationary distribution $\pi_{\psi}$, a fault diagnosis scheme is launched. As we have already seen $\pi_{\psi}$ allows the computation of a consensus transition matrix $\mathbf{P}_{c}$ as a weighted sum of the unobservable Markov chains of all agents in the group; see (5). Computing the stationary distribution $\pi_{c}$ of a consensus transition matrix $\mathbf{P}_{c}$ is the starting point of Algorithm 1. A practical implementation of line 1 of the algorithm is slightly modified in that the condition $\delta\left(\pi_{c}, \pi_{P_{i}^{(0)}}\right)>0$ is tested as $\log \delta\left(\pi_{c}, \pi_{P_{i}^{(0)}}\right)<\kappa$, for suitable $\kappa$, assumed here to be $\kappa=-20$. In Fig. 9 the logarithm of the distance measure of all agents the group is given, showing how it changes over time.

In view of Fig. 9 notice first that in the interval $t \in[103,303]$ all three agents assessed the value $\log \delta\left(\pi_{c}, \pi_{P_{i}^{(0)}}\right), i=1,2,3$, as being less than the threshold $\kappa$, indicating faults in the 
TABLE VIII

Model Stationary Distributions

$$
\begin{array}{l|ll}
\pi_{\boldsymbol{A}_{1}} & 0.3846 & 0.6154 \\
\hline \pi_{\boldsymbol{A}_{2}} & 0.5556 & 0.4444 \\
\hline \pi_{\boldsymbol{A}_{3}} & 0.5035 & 0.4965
\end{array}
$$

TABLE IX

Consensus Stationary Distributions at times $t=50,150,250,350$

$$
\begin{array}{l|ll}
\pi_{c}^{t=50} & 0.3846 & 0.6154 \\
\hline \pi_{c}^{t=150} & 0.4804 & 0.5196 \\
\hline \pi_{c}^{t=250} & 0.5015 & 0.4985 \\
\hline \pi_{c}^{t=350} & 0.3846 & 0.6154
\end{array}
$$

system. Secondly, it is evident that agent 2 has slightly higher fluctuations on the interval $t=[204,303]$ than other agents. Before we give a detailed explanation of the reasons for this behaviour, let us look at both the value of the stationary distributions $\pi_{\boldsymbol{A}_{i}}$ of models $\lambda\left(\Theta_{i}\right), i=1,2,3$ (Table VIII) and the consensus matrices $\pi_{c}$ at times $t=50,150,250,350$ (Table IX). We see that that $\pi_{\boldsymbol{A}_{3}}$ and $\pi_{c}^{t=250}$ have similar values. From (5) it follows that consensus transition matrix cannot be equal to any particular initially pooled transition matrix, except in the trivial case that all transition matrices are the same. However, in any non-trivial case, a problem of similarity between unobservable Markov chains arises, indicating the importance of measuring it in the training phase. As proposed by Rabiner [33] the concept of model distance can be used for this purpose. This problem will be the subject of future research.

Fig. 10 illustrates how the group members perceive group behaviour and how they achieve behavioural consensus; exchanging model parameters among group members and by applying local observations to these models leads to a common perception of group behaviour. 

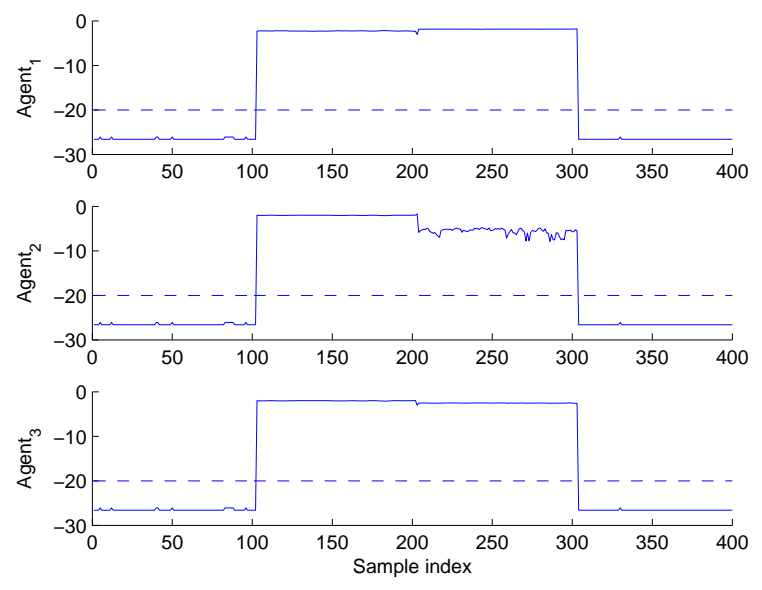

Fig. 9. Distribution Distance
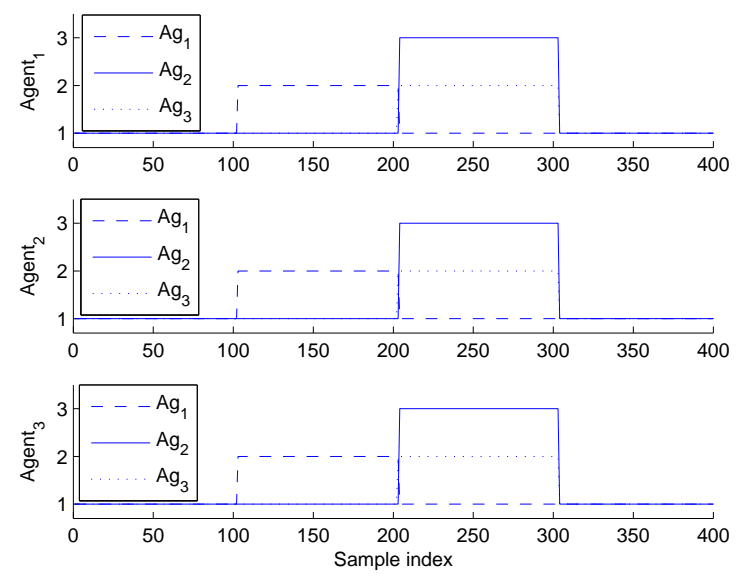

Fig. 10. Group Decision

\section{Discussion}

We have proposed a fault detection scheme for distributed systems in which subsystems are represented by agents. Operating modes of subsystems are modelled by Markov chains. The agents form groups whose common (consensus) transition matrix is estimated. Change in consensus within the group is monitored and, once a change is detected, the distances between the stationary distributions of operating modes are estimated in order to identify 
the new condition of the system. Future work will include the practical implementation of our algorithm to fault diagnosis in power systems. To be fully applicable it will be necessary to extend our approach to accommodate continuous observation schemes.

\section{ACKNOWLEDGments}

This work was supported by the Australian Research Council Centre of Excellence for Mathematics and Statistics of Complex Systems (MASCOS). We are grateful to Ross McVinish for helpful comments.

\section{REFERENCES}

[1] R. D. Leitch, Reliability analysis for engineers : an introduction. New York : Oxford University Press, 1995.

[2] R. Patton, P. Frank, and R. Clark, Eds., Issues of fault diagnosis for dynamic systems. London : New York: Springer, 2000.

[3] S. X. Ding, Model-based fault diagnosis techniques: design schemes, algorithms, and tools. Berlin, Heidelberg : Springer-Verlag Berlin Heidelberg, 2008.

[4] L. H. C. E. Russell and R. D. Braatz, Data-driven methods for fault detection and diagnosis in chemical processes. New York : Springer, 2000.

[5] J. Gertler, Fault detection and diagnosis in engineering systems. Marcel Dekker, Inc., 1998.

[6] S. Simani, C. Fantuzzi, and R. Patton, Model-based fault diagnosis in dynamic systems using identification techniques. London; New York : Springer, 2003.

[7] R. Isermann, "Model base fault detection and diagnosis methods," in American Control Conference, 1995. Proceedings of the, vol. 3, Jun 1995, pp. 1605-1609.

[8] — - "Process fault detection based on modeling and estimation methodsŮa survey," Automatica, vol. 20, no. 4, pp. $387-404,1984$.

[9] —_ "Model-based fault-detection and diagnosis - status and applications," Annual Reviews in Control, vol. 29, no. 1, pp. 71-85, 2005.

[10] D. Siljak, Decentralized control of complex systems. Academic Press, 1991.

[11] E. Fabre and V. Pigourier, "Monitoring distributed systems with distributed algorithms," in Decision and Control, 2002, Proceedings of the 41st IEEE Conference on, vol. 1, 2002, pp. 411 - 416 vol.1.

[12] R. Setola and S. De Porcellinis, "Complex networks and critical infrastructures," in Modelling, Estimation and Control of Networked Complex Systems, ser. Understanding Complex Systems, A. Chiuso, L. Fortuna, M. Frasca, A. Rizzo, L. Schenato, and S. Zampieri, Eds. Springer Berlin / Heidelberg, 2009, vol. 50, pp. 91-106.

[13] R. E. Kalman and R. S. Bucy, "New results in linear filtering and prediction theory," Journal of Basic Engineering, vol. 83, no. 1, pp. 95-108, 1961.

[14] J. R. Norris, Markov chains. Cambridge ; New York : Cambridge University Press, 1997.

[15] M. Stone, "The opinion pool," The Annals of Mathematical Statistics, vol. 32, no. 4, pp. 1339-1342, 1961.

[16] M. H. DeGroot, "Reaching a consensus," Journal of the American Statistical Association, vol. 69, no. 345, pp. $118-121,1974$. 
[17] S. Chatterjee and E. Seneta, "Towards consensus: Some convergence theorems on repeated averaging," Journal of Applied Probability, vol. 14, no. 1, pp. pp. 89-97, 1977.

[18] E. Franco, R. Olfati-Saber, T. Parisini, and M. Polycarpou, "Distributed fault diagnosis using sensor networks and consensus-based filters," 2006, pp. 386-391.

[19] R. Ferrari, T. Parisini, and M. Polycarpou, "Distributed fault diagnosis with overlapping decompositions and consensus filters," in American Control Conference, 200\%. ACC '0\%, 2007, pp. 693 -698.

[20] S. Stankovic, N. Ilic, M. Stankovic, and K. Johansson, "Distributed change detection based on a consensus algorithm," Signal Processing, IEEE Transactions on, vol. 59, no. 12, pp. 5686 -5697, 2011.

[21] A. Aghasaryan, E. Fabre, A. Benveniste, R. Boubour, and C. Jard, "Fault detection and diagnosis in distributed systems: An approach by partially stochastic petri nets," Discrete Event Dynamic Systems, vol. 8, pp. 203-231, 1998.

[22] A. Benveniste, E. Fabre, and S. Haar, "Markov nets: probabilistic models for distributed and concurrent systems," Automatic Control, IEEE Transactions on, vol. 48, no. 11, pp. 1936 - 1950, 2003.

[23] T. Kato, H. Kanamori, Y. Suzuoki, and T. Funabashi, "Multi-agent based control and protection of power distributed system - protection scheme with simplified information utilization -," in Intelligent Systems Application to Power Systems, 2005. Proceedings of the 13th International Conference on, 2005, pp. 49 -54.

[24] L. Garza, F. Cantu, and S. Acevedo, "Integration of fault detection and diagnosis in a probabilistic logic framework," in Advances in Artificial Intelligence, IBERAMIA 2002, F. Garijo, J. Riquelme, and M. Toro, Eds. Springer Berlin Heidelberg, 2002, vol. 2527, pp. 265-274.

[25] C. Bron and J. Kerbosch, "Algorithm 457: finding all cliques of an undirected graph," Commun. ACM, vol. 16, pp. 575-577, 1973.

[26] R. A. Horn and C. R. Johnson, Topics in matrix analysis. Cambridge ; Melbourne : Cambridge University Press, 1991.

[27] A. N. Langville and W. J. Stewart, "The kronecker product and stochastic automata networks," Journal of Computational and Applied Mathematics, vol. 167, no. 2, pp. 429 - 447, 2004.

[28] T. Batu, S. Guha, and S. Kannan, "Inferring mixtures of markov chains," National Institute of Standards and Technology, Tech. Rep., 2002.

[29] H. Frydman, "Estimation in the Mixture of Markov chains," SSRN eLibrary, 2003.

[30] W. J. Stewart, Introduction to the numerical solution of Markov chains. Princeton, N.J. : Princeton University Press, 1994.

[31] T. W. Anderson and L. A. Goodman, "Statistical inference about markov chains," The Annals of Mathematical Statistics, vol. 28, no. 1, pp. 89-110, 1957.

[32] A. P. Dempster, N. M. Laird, and D. B. Rubin, "Maximum likelihood from incomplete data via the em algorithm," Journal of the Royal Statistical Society. Series B (Methodological), vol. 39, no. 1, pp. 1-38, 1977.

[33] L. Rabiner, "A tutorial on hidden markov models and selected applications in speech recognition," Proceedings of the IEEE, vol. 2, pp. 257-286, 1989.

[34] O. Cappé, E. Moulines, and T. Ryden, Inference in Hidden Markov Models. Springer, 2005.

[35] A. Viterbi, "Error bounds for convolutional codes and an asymptotically optimum decoding algorithm," Information Theory, IEEE Transactions on, vol. 13, no. 2, pp. 260-269, 1967. 
[36] L. E. Baum and T. Petrie, "Statistical inference for probabilistic functions of finite state markov chains," The Annals of Mathematical Statistics, vol. 37, no. 6, pp. 1554-1563, 1966. 